\title{
Value of prophylactic antibiotics for invasive dental procedures unclear
}

\section{Abstracted from \\ Glenny AM, Oliver R, Roberts GJ, Hooper L, Worthington HV.}

Antibiotics for the prophylaxis of bacterial endocarditis in dentistry. Cochrane Database of Syst Rev 2013; 10: Art. No: CD003813. DOI: 10.1002/14651858.CD003813.pub4.

Address for correspondence: Luisa Fernandez Mauleffinch, Review Group Co-ordinator, Cochrane Oral Health Group, MANDEC, School of Dentistry, University of Manchester, Higher Cambridge Street, Manchester, M15 6FH, UK. E-mail: luisa.fernandez@manchester.ac.uk.

\section{Question: Are prophylactic antibiotics required before invasive dental procedures in people at risk or at high risk of bacterial endocarditis?}

Data sources The Cochrane Oral Health Group's Trials Register, the Cochrane Central Register of Controlled Trials (CENTRAL), Medline, Embase, the US National Institutes of Health Trials Register and the metaRegister of Controlled Trials.

Study selection Randomised controlled trials (RCTs) and controlled clinical trials (CCTs) would be included where available. Due to the low incidence of bacterial endocarditis it was anticipated that few such trials would be found. Cohort and case-control studies were included where suitably matched control or comparison groups had been studied. Data extraction and synthesis Two review authors independently selected studies for inclusion then assessed risk of bias and extracted data from the included study.

Results Only one case controlled study met the inclusion criteria. It collected all the cases of endocarditis in the Netherlands over two years, finding a total of 24 people who developed endocarditis within 180 days of an invasive dental procedure, definitely requiring prophylaxis according to current guidelines, and who were at increased risk of endocarditis due to a pre-existing cardiac problem. This study included participants who died because of the endocarditis (using proxies). Controls attended local cardiology outpatient clinics for similar cardiac problems, had undergone an invasive dental procedure within the past 180 days, and were matched by age with the cases. No significant effect of penicillin prophylaxis on the incidence of endocarditis could be seen. No data were found on other outcomes.

Conclusions There remains no evidence that antibiotic prophylaxis is either effective or ineffective against bacterial endocarditis in people considered at risk who are about to undergo an invasive dental procedure. It is not clear whether the potential harms and costs of penicillin administration outweigh any beneficial effect. Ethically, practitioners need to discuss the potential benefits and harms of antibiotic prophylaxis with their patients before a decision is made about administration.

This paper is based on a Cochrane Review published in the Cochrane Library 2013, issue 10 (see www.thecochranelibrary.com for information). Cochrane Reviews are regularly updated as new evidence emerges and in response to feedback, and the Cochrane Library should be consulted for the most recent version of the review.

\section{Commentary}

The recommendation for antibiotic prophylaxis prior to dental procedures for patients at high risk for bacterial endocarditis is a controversial topic worldwide and has been under debate for the last few decades.

Evidence-based research was used to revise older guidelines, allowing practitioners and patients vital information to make informed decisions regarding antibiotic prophylaxis. Part of the information included in the revision addresses the rare but possible anaphylaxis response to the single dose antibiotic.

This systematic review was published after the American Heart Association (AHA) ${ }^{1}$ published their reviewed guidelines in 2007 and the National Institute for Health and Care Excellence (NICE) in the UK revised their guidelines in 2008 for prevention of infective bacterial endocarditis. ${ }^{2}$ This systematic review used appropriate search strategies in that they accepted case controlled studies in their inclusion criteria. A randomised clinical trial would be questionable to carry out in some parts of the world since giving or not giving antibiotics to prevent bacterial endocarditis in vulnerable patients may result in a fatal outcome.

As a Cochrane review the search, selection of articles and the critical appraisal were done by more than one reviewer and followed the correct protocols on conducting a systematic review. One article selected for final discussion was a case control study done in 1992. This study was conducted in the Netherlands and was assessed for bias and quality, which was referenced in the AHA and NICE guidelines.

Antibiotic prophylaxis is still a polemic topic in dentistry. For many years it was believed that bacterial endocarditis was caused by the introduction of oral bacteria systemically and for those patients that had certain cardiac conditions the risk was high. As a result, premedication with antibiotic prophylaxis was required prior to undergoing invasive dental therapy.

The American Heart Association (AHA) revised those recommendations and many of the previously pre-medicated conditions were no longer considered for antibiotic coverage prior to dental treatment.

Interestingly, The National Institute for Health and Care Excellence (NICE) and the American Heart Association differ in their recommendations for antibiotic premedication. The AHA recommends that certain conditions be pre-medicated, whereas NICE determined that no antibiotic coverage is needed prior to any dental procedure.

A study in England, which analysed the impact of the NICE guidelines specifically, looked at antibiotic pre-medication for 
high-risk patients undergoing invasive dental procedures. They examined the incidence of infective endocarditis and concluded that there was no significant increase in the number of cases. The authors suggested that ongoing data monitoring is needed to determine if antibiotic prophylaxis has a role in protecting a small group of patients at higher risk. ${ }^{3}$

The challenge each practitioner encounters is the interpretation of the evidence obtained and its clinical application. Adopting a protocol to follow is further complicated by the use of different guidelines in other parts of the world. In addition to invasive dental procedures as a risk for bacterial endocarditis, we need to consider daily activities such as eating and oral hygiene practices among those factors. This further supports our role in advocating good oral home care.

\section{Glenn K Rochlen and Analia Veitz Keenan} NYU College of Dentistry, New York, USA

1. Wilson W, Taubert KA, Gewitz M, et al. AHA Guideline: Prevention of Infective Endocarditis: Guidelines From the American Heart Association: A Guideline From the American Heart Association Rheumatic Fever, Endocarditis, and Kawasaki Disease Committee, Council on Cardiovascular Disease in the Young, and the Council on Clinical Cardiology, Council on Cardiovascular Surgery and Anesthesia, and the Quality of Care and Outcomes. Circulation 2007; 116: 1736-1754. Published online before print April 19 2007, doi:10.1161/CIRCULATIONAHA.106.183095

2. Prophylaxis Against Infective Endocarditis: Antimicrobial Prophylaxis Against Infective Endocarditis in Adults and Children Undergoing Interventional Procedures. CG64 National Institute for Health and Care Excellence London: 2008

3. Thornhill MH, Dayer MJ, Forde JM, et al. Impact of the NICE guideline recommending cessation of antibiotic prophylaxis for prevention of infective endocarditis: before and after study. BMJ 2011; 342: d2392. doi: 10.1136/bmj.d2392

Evidence-Based Dentistry (2014), 15, 12-13. doi: 10.1038/sj.ebd.6400983 